\title{
Efficient unidirectional polarization-controlled excitation of surface plasmon polaritons
}

\begin{abstract}
Anders Pors ${ }^{1, *}$, Michael G Nielsen ${ }^{1,2, *}$, Thomas Bernardin ${ }^{2}$, Jean-Claude Weeber ${ }^{2}$ and Sergey I Bozhevolnyi ${ }^{1}$
Efficient excitation of surface plasmon polaritons (SPPs) remains one of the most challenging issues in areas of plasmonics related to information communication technologies. In particular, combining high SPP excitation efficiency and acceptance of any polarization of incident light appeared to be impossible to attain due to the polarized nature of SPPs. Here we demonstrate plasmonic couplers that represent arrays of gap SPP resonators producing upon reflection two orthogonal phase gradients in respective linear polarizations of incident radiation. These couplers are thereby capable of efficiently converting incident radiation with arbitrary polarization into SPPs that propagate in orthogonal directions dictated by the phase gradients. Fabricated couplers operate at telecom wavelengths and feature the coupling efficiency of $\sim \mathbf{2 5 \%}$ for either of two linear polarizations of incident radiation and directivity of SPP excitation exceeding 100. We further demonstrate that an individual wavelength-sized unit cell, representing a meta-scatterer, can also be used for efficient and polarization sensitive SPP excitation in compact plasmonics circuits.
\end{abstract}

Light: Science \& Applications (2014) 3, e197; doi:10.1038/lsa.2014.78; published online 15 August 2014

Keywords: gap surface plasmons; metamaterials; metasurfaces; surface plasmon polaritons

\section{INTRODUCTION}

Surface plasmon polaritons (SPPs) are electromagnetic excitations, in which electromagnetic field in dielectric are coupled to collective electron oscillations in metal, which propagate along and are tightly bound to metal/dielectric interfaces. ${ }^{1}$ Modern plasmonics, which embraces various phenomena associated with excitation, propagation and scattering of SPPs, became ubiquitous in extremely diverse areas, ranging from biochemical sensing, ${ }^{2}$ quantum optics ${ }^{3}$ and information communication technologies ${ }^{4}$ to sustained energy sources. ${ }^{5}$ SPPs are essentially transverse magnetic waves with the magnetic field oriented perpendicular to the propagation plane, a very important feature that dictates the polarization sensitivity of the SPP excitation efficiency by free propagating radiation. It is therefore understandable that various SPP couplers developed in the quest for the efficient and unidirectional SPP excitation operate with only one (linear) polarization of the incident light, ${ }^{6-10}$ resulting thereby in the loss of light power carried by the orthogonal polarization.

The recent progress in optical metasurfaces, which influence transmitted and reflected optical fields by imposing additional (surface) gradients onto their phases, ${ }^{11,12}$ opened new possibilities for efficient coupling of propagating and surface waves. ${ }^{13,14}$ Very recently, polarization-controlled tunable directional SPP coupling has been demonstrated using arrays of narrow (elongated) apertures in an otherwise opaque metal film, so that the direction of SPP excitation was dictated by the helicity of a circularly polarized incident beam. ${ }^{15,16}$ Note that, in these configurations, the SPP excitation involves both the transmission through narrow apertures and the coupling of the transmitted radiation to SPPs, so that the overall coupling efficiency is very difficult to evaluate, and, so far, it has not been quantified. ${ }^{15,16}$ At the same time, gap surface plasmon (GSP)-based gradient metasurfaces, in which arrays of rectangular metal patches are placed on a thin dielectric layer supported by a thick metal film or substrate, offer very high (close to $100 \%$ ) efficiency when operating with free propagating waves. ${ }^{17-19}$ The presence of a continuous metal support in this configuration makes it also naturally compatible and, thereby, very attractive for implementing efficient SPP excitation. ${ }^{20}$

Here, we realize efficient unidirectional polarization-controlled SPP excitation by applying our approach of independently manipulating orthogonal polarizations of reflected light with GSP-based gradient metasurfaces ${ }^{21}$ to the SPP excitation. In particular, we design arrays of GSP resonators that would produce upon reflection two orthogonal phase gradients in two respective linear polarizations of incident radiation, so that the incident radiation (with arbitrary polarization) can efficiently (numerically estimated up to $40 \%$ ) be converted into SPPs propagating in orthogonal directions dictated by the phase gradients. Using 50-nm-thick dielectric spacer layers covering 80 -nm-thick gold films supported by glass substrates, we fabricate GSP-based coupling arrays that operate at telecom wavelengths and feature the coupling efficiency of $\sim 25 \%$ for either of two linear polarizations of incident radiation and the directivity of SPP excitation exceeding 100. We further demonstrate that an individual wavelength-sized unit cell, representing a meta-scatterer, can also be used for efficient and polarization sensitive SPP excitation in compact plasmonics circuits.

${ }^{1}$ Department of Technology and Innovation, University of Southern Denmark, Odense, Denmark and ${ }^{2}$ LICB, UMR 6303 CNRS - University of Burgundy, F-21078 Dijon, France *These authors contributed equally to this work

Correspondence: Professor SI Bozhevolnyi, Department of Technology and Innovation, University of Southern Denmark, Niels Bohrs Allé 1, DK-5230 Odense M, Denmark E-mail: seib@iti.sdu.dk

Received 23 November 2013; revised 7 May 2014; accepted 13 May 2014 


\section{MATERIALS AND METHODS}

Configurations explored in this work for efficient unidirectional polarization-controlled SPP excitation represent GSP-based metasurfaces ensuring the appropriate phase gradients. The design procedure is somewhat similar to that described in our previous work on total control of reflected light with GSP-based metasurfaces, ${ }^{21}$ but also substantially different, because the structure period is, in the present case, determined by the corresponding SPP wavelength rather than by the angle of diffraction into free space. Let us first consider the problem of efficient unidirectional SPP excitation with one-dimensional (1D) GSP-based metasurfaces, which create the appropriate phase gradient only in one direction. In this case, GSP resonator arrays can be formed with long metal stripes placed atop a dielectric spacer supported by a thick metal film (Figure 1a), forming an array of continuous-layer GSP resonators. ${ }^{22}$ For normal incidence of the radiation at the design wavelength $\lambda=1500 \mathrm{~nm}$ and a 50-nm-thick glass film atop a thick gold layer, the SPP wavelength and thereby the super-cell period is estimated to be $1467 \mathrm{~nm}$. In order to avoid nm-sized strip widths and gaps, which might be too demanding from the fabrication point of view, we consider three 489-nm-wide cells constituting a 1467-nm-wide super cell and calculate the complex reflection coefficient for transverse magnetic-polarized light (in which the electric field is perpendicular to the strip direction) as a function of the width $w$ of 50-nm-thin gold strips placed on the top of the glass-gold sandwich in an infinitely extended 489-nm-period strip array (Figure 1b). It is seen that the reflected light phase varies rapidly in the vicinity of the GSP resonance at $w \cong 280 \mathrm{~nm}$, resulting in a sufficiently large phase range to produce the phases needed for the three-cell coupling structure, i.e., $-2 \pi / 3,0$ and $2 \pi / 3$. These reflection phases are represented by the strip widths marked by vertical dotted lines in Figure $1 \mathrm{~b}$. The choice of the 1467-nm-wide super cell to be composed of three 489-nm-wide cells, each containing a strip with one of the three marked widths, is based on the assumption that the individual response phase is only weakly influenced by the widths of neighbouring strips, an assumption that has been verified in our previous investigations $^{21}$ and used throughout this work. Efficient unidirectional SPP excitation in the direction determined by the incident polarization (i.e., polarization-controlled SPP excitation) requires the use of two-dimensional (2D) arrays of GSP resonators forming birefringent metasurfaces ${ }^{21}$ with orthogonal linear phase gradients for two polarizations and a super cell periodicity equal to the SPP wavelength.

As a final comment, it should be mentioned that in the design of SPP couplers, being either 1D- or 2D-periodic, we disregard variations in reflection amplitudes produced by different elements comprising the super cell (Figures $1 \mathrm{~b}$ and $2 \mathrm{~b}$ ). Although one should ideally strive to achieve constant reflection amplitude (with a value close to one) in order to maximize the SPP coupling efficiency and directionality of SPP excitation, the possibility to simultaneously control both the reflection phase and amplitude requires the usage of more complex unit cells, with a larger number of geometrical parameters to vary, hence complicating both the design and fabrication procedures. At the same time, the equivalence in operation of gradient metasurfaces and blazed gratings suggests that the presented approach is robust against design and fabrication imperfections ${ }^{23}$ - an important feature that we have experimentally verified for birefringent metasurfaces. ${ }^{21}$

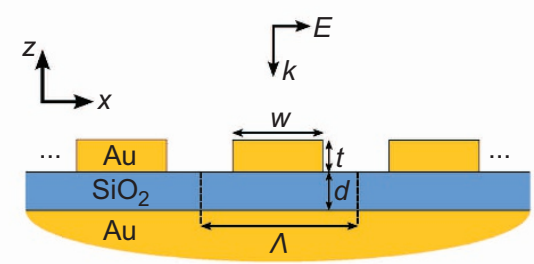

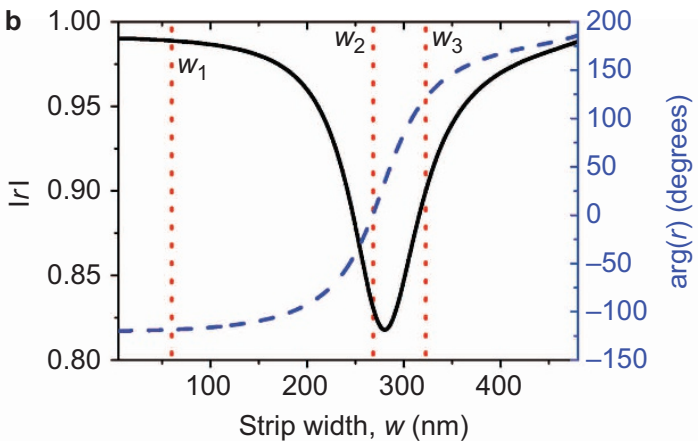

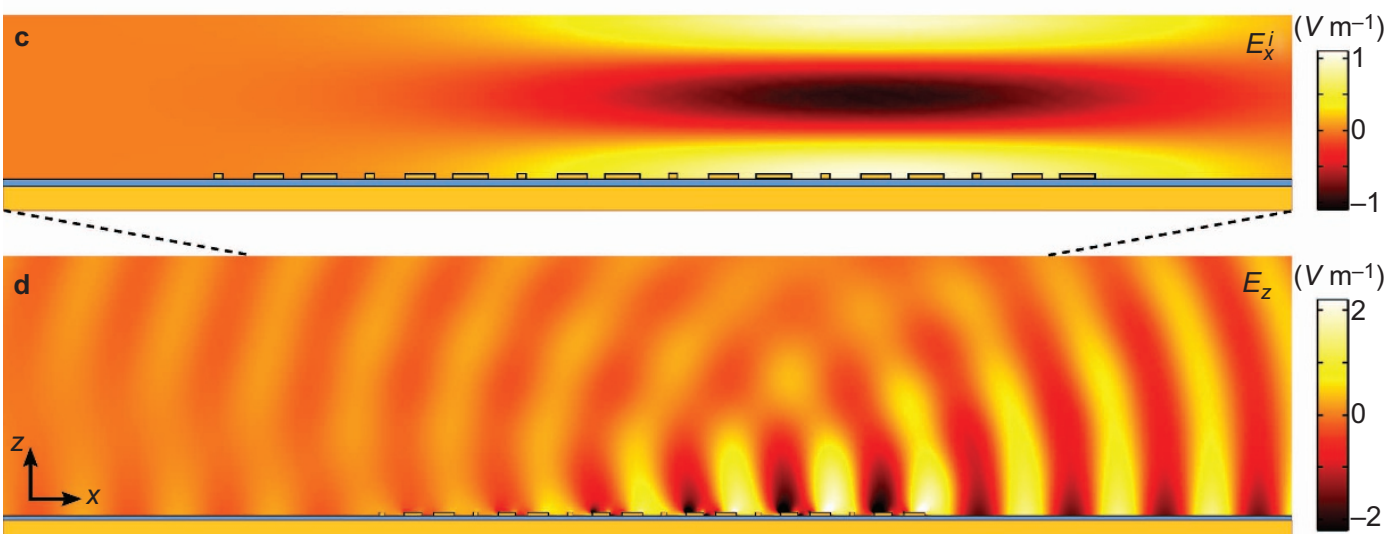

Figure 1 Design of 1D couplers for efficient and unidirectional SPP excitation. (a) Sketch of 1D-periodic GSP-based metasurface. The incident field is TM-polarized (i.e., $x$-polarized) and propagates normal to the surface. (b) Amplitude and phase of reflected light from metasurface in (a) as a function of strip width $w$ when $\lambda=1500 \mathrm{~nm}, \Lambda=489 \mathrm{~nm}$ and $d=t=50 \mathrm{~nm}$. Vertical dotted lines indicate the three chosen strip widths (with phase difference of $2 \pi / 3$ ) constituting a super cell in the SPP coupler. The time convention is $\exp (-\mathrm{i} \omega t)$. (c) Electric field of the incident $x$-polarized Gaussian beam overlaid by a sketch of the SPP coupler. The SPP coupler consists of six super cells, corresponding to an overall size of $L_{c}=18 \Lambda=8.8 \mu \mathrm{m}$, and the Gaussian beam is centred at $x_{0}=0.74 L_{c}$ with a beam radius of $w_{0}=3 \mu \mathrm{m}$. (d) $z-$ component of the electric field, corresponding to the transverse electric field component of SPPs. 1D, one-dimensional; GSP, gap surface plasmon; SPP, surface plasmon polariton; TM, transverse magnetic. 


\section{RESULTS AND DISCUSSION}

In the following, we first numerically study a realistic 1D-periodic SPP coupler consisting of six super cells being illuminated by a normal incident Gaussian beam with a $3-\mu \mathrm{m}$ radius and positioned so as to maximize the excitation efficiency of SPPs propagating in the $+x$ direction (Figure 1c). The choice of six super cells results from the demand of compact SPP couplers and numerical simulations demonstrating no appreciable gain in excitation efficiency for larger couplers. The SPP coupler is characterized by the coupling efficiency $C$, representing the amount of incident power being coupled to SPPs propagating in the $+x$-direction, and the directivity $D$, describing the ratio of SPP power flowing in the $+x$ - and $-x$-direction, which in our case amounts in $C \cong 40 \%$ and $D \cong 53$ (for further details on the calculation procedure, see Supplementary Information). This high directivity is in agreement with visual observation (Figure 1d), confirming that the off-centre positioning of the input beam together with the appropriate quasi-linear phase gradient in the reflected (by the SPP coupler strip structure) field result in the SPP excitation predominantly in the $+x$-direction. One should note that the performance of the SPP coupler is inherently limited due to the circumstance that SPP fields excited at one part of the coupler propagate along and interact with other parts. ${ }^{14}$ The excited SPPs become thereby partially absorbed in the GSP resonators (constituting the coupler) and coupled out (by the coupler grating structure) into free propagating waves. Additionally, the wavelength of SPPs in the coupler region is slightly smaller compared to the region outside, thus indicating a non-optimal phasematching condition for the chosen super cell period. Taking the above comments into account, it is apparent that the performance of the SPP coupler can be further improved by iteratively changing the unit cell period and strip widths. In fact, by changing the unit cell period $\Lambda$ from $489 \mathrm{~nm}$ to $468 \mathrm{~nm}$, the second strip width $w_{2}$ from $268 \mathrm{~nm}$ to $255 \mathrm{~nm}$, and the third strip width $w_{3}$ from $323 \mathrm{~nm}$ to $360 \mathrm{~nm}$, the resulting numerical coupling efficiency reaches $C \cong 47 \%$ with an extremely large directivity of $D \cong 3215$. Note that a small change in the width of the first strip ( $w_{1}=60 \mathrm{~nm}$ ) has no effect on the coupling efficiency, as the strip is far away from the resonance region (Figure 1b).

We now consider the design of polarization-controlled 2D-periodic SPP couplers, which relies on the design of GSP-based metasurfaces ${ }^{21}$ with orthogonal linear phase gradients for two orthogonal polarizations and a super cell periodicity equal to the SPP wavelength (assuming normal incident light). The starting point is a homogeneous metasurface, whose basic unit cell consists of a gold nanobrick atop a 50-nmthick glass spacer and a thick gold substrate, illuminated by a normal incident plane wave that is either $x$ - or $y$-polarized (Figure 2a). Here, the nanobrick dimensions $L_{x}$ and $L_{y}$ represent two parameters that one can vary to independently control the phase of the reflected light for the two orthogonal polarizations. For the design wavelength $\lambda=1500 \mathrm{~nm}$ and geometrical parameters following those of the 1D-periodic SPP coupler, Figure $2 \mathrm{~b}$ displays the complex reflection coefficient of the homogenous metasurface as a function of $L_{x}$ and $L_{y}$. It is seen that away from the GSP resonance, which occurs at $L_{x} \sim 300 \mathrm{~nm}$ and $L_{y} \sim 50 \mathrm{~nm}$ for $x$-polarized light (and correspondingly at $L_{x} \sim 50 \mathrm{~nm}$ and $L_{y} \sim 300 \mathrm{~nm}$ for $y$-polarized light), the reflection phase is primarily influenced by the nanobrick dimension in the direction of light polarization, resulting in the constant-phase lines being practically parallel to the corresponding axes. One should also note that, e.g., for $x$-polarized incident light, the reflection (phase and amplitude) as a function of $L_{x}$ approaches the reflection from the nanostrip metasurface (Figure 1b) when the other
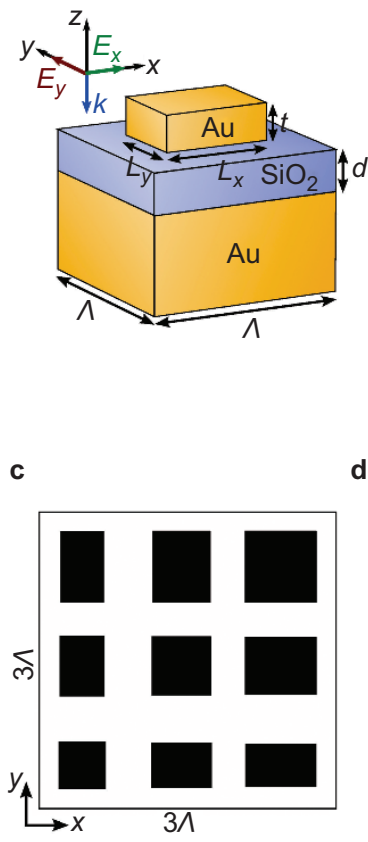

b
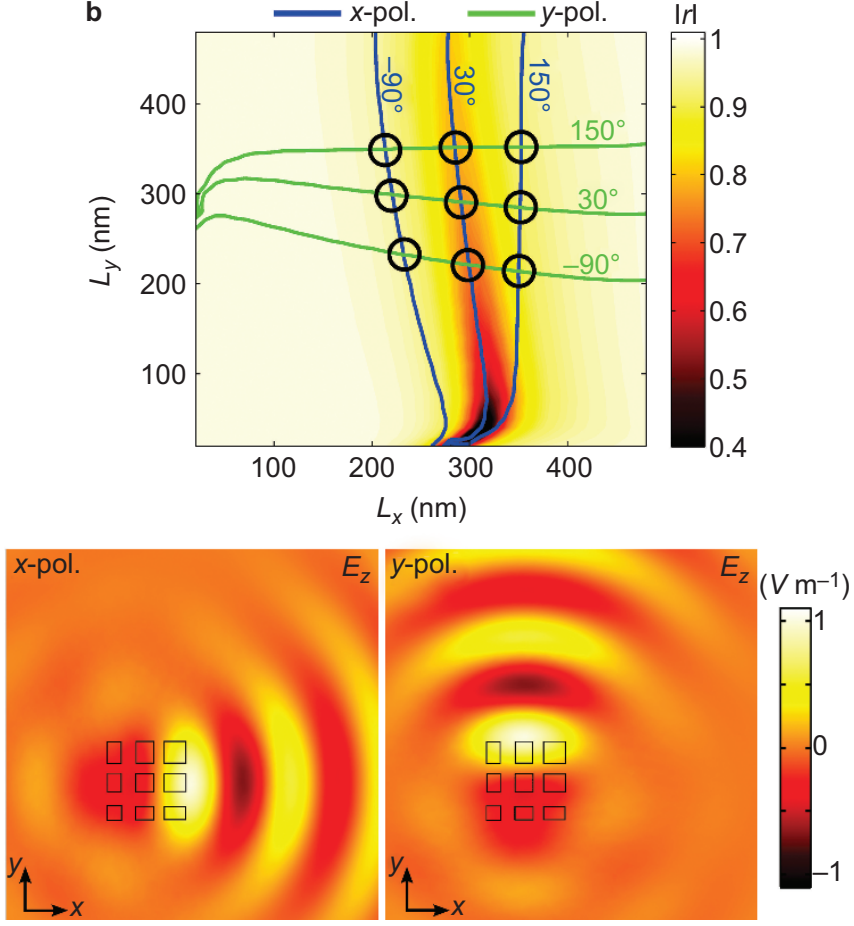

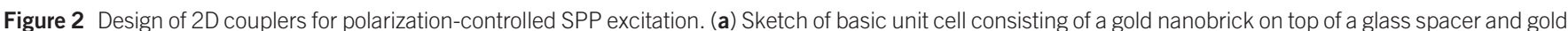

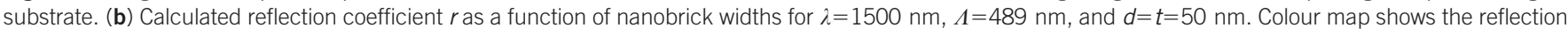

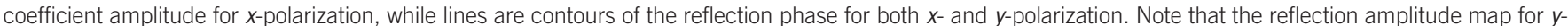

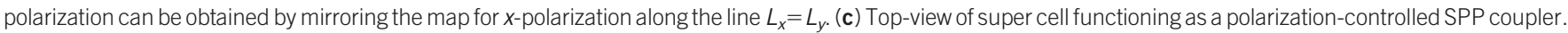

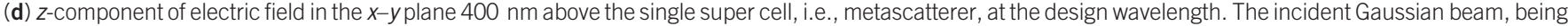
either $x$ - or $y$-polarized, is positioned at the centre of the metascatterer with a beam radius of $w_{0}=2 \mu \mathrm{m}$. 2D, two-dimensional; SPP, surface plasmon polariton. 
dimension, $L_{y}$, comes close to the unit cell size $\Lambda$, thus indicating the transition from nanobrick to nanostrip metasurfaces. The reflection phase for both $x$ - and $y$-polarization is discretized into three constantphase contour lines with a step of $2 \pi / 3$, thereby allowing us to visually determine the nine appropriate nanobrick dimensions corresponding to the intersections marked with circles. Accordingly, the polarizationcontrolled SPP coupler (with orthogonal linear phase gradients for $x$ and $y$-polarization) can be constructed by placing the nine elements with a centre-to-centre distance of $\Lambda=489 \mathrm{~nm}$ into a $3 \times 3$ super cell (Figure 2c). Ideally, one would like to numerically investigate the performance of a SPP coupler consisting of $6 \times 6$ super cells. However, such a calculation is too computationally demanding in our current hardware set-up, thus forcing us to make an alternative two-step evaluation. In the first step, we verify that SPPs are excited unidirectionally and orthogonally to each other for orthogonal polarizations by modelling an $x$ - and $y$-polarized Gaussian beam impinging on a single super cell (Figure $2 \mathrm{~d}$ ). In the second step, we estimate the coupling efficiency and directivity of the $6 \times 6$ SPP coupler by treating each row of the super cell (Figure 2c) as its own super cell in which we have $\Lambda$-sized periodicity in the $y$-direction and six super cells in the $x$-direction. By illuminating the SPP coupler with an off-centred $x$-polarized Gaussian beam with beam radius $w_{0}=3 \mu \mathrm{m}$, we calculate for the three different cases (i.e., rows) coupling efficiencies and directivities in the range $C \cong 41 \%-43 \%$ and $D \cong 56-60$, respectively. One should note that the symmetry of the $3 \times 3$ super cell (Figure $2 \mathrm{c}$ ) ensures identical performance when considering the columns of the super cell and $y$-polarization. For this reason, we can conclude that the $6 \times 6$ polarization-controlled SPP coupler with a coupling efficiency of $\sim 40 \%$ and directivity of $\sim 50$ performs very similar to the non-optimized 1D-periodic counterpart previously discussed.

With the above numerical simulations illustrating the possibility for efficient unidirectional excitation of SPPs, including polarization-controlled excitation using 2D-periodic couplers, we now move on to the experimental verification. Figure 3 displays images of the lithographically fabricated SPP couplers consisting of 6 and $6 \times 6$ super cells for the $1 \mathrm{D}$ - and 2D-periodic cases (Figure $3 \mathrm{a}$ and $3 \mathrm{~b}$ ), respectively, together with a sketch of the leakage radiation microscopy (LRM) set-up used for real-time imaging (integration time $\sim 100 \mu \mathrm{s}$ ) of excited SPPs (Figure 3c; for further details, see Supplementary Information). Using a linearly-polarized Gaussian input beam with $1 / \mathrm{e}^{2}$ spot size $w_{0}=2.8 \pm 0.3 \mu \mathrm{m}$ and positioned for optimal coupling efficiency, Figure $4 \mathrm{a}$ shows the corresponding LRM image of the 1D-periodic SPP coupler at the design wavelength $\lambda=1500 \mathrm{~nm}$, verifying the unidirectional excitation of SPPs. The LRM image is further analysed in order to evaluate the SPP propagation length $L_{\mathrm{p}}$, coupling efficiency and directivity. For example, the propagation length, which is used in the calculation of the coupling efficiency, can be estimated by evaluating the LRM intensity at cross-cuts taken along the $y$-direction as a function of the displacement $u=\left|x-x_{0}\right|$, where $x_{0}$ is the $x$-coordinate of the excitation spot. By fitting a Gaussian function to each cross-cut, we obtain the SPP intensity $I_{x}$ and the SPP beam waist $w_{x}$ at distances $u$. The product $I_{x} w_{x}$ decreases exponentially as a function of distance $u$ (Figure $4 \mathrm{~b}$ ) and immediately gives access to the SPP propagation length by fitting a first order polynomial $\ln \left(I_{x} w_{x}\right)=\ln (A)-u / L_{\mathrm{p}}$, where $A$ is a constant, to the recorded data points. In particular, the experimentally evaluated propagation lengths of about $85-90 \mu \mathrm{m}$ are in reasonable agreement with the theoretical propagation length of $\sim 110 \mu \mathrm{m}$. The coupling efficiency is a bit more cumbersome to evaluate, so here we simply state the result, delegating the calculation details to the Supplementary Information. Thus, we obtain a coupling efficiency of $\sim 27 \% \pm 4 \%$, which is somewhat lower than theoretically predicted value of $47 \%$.
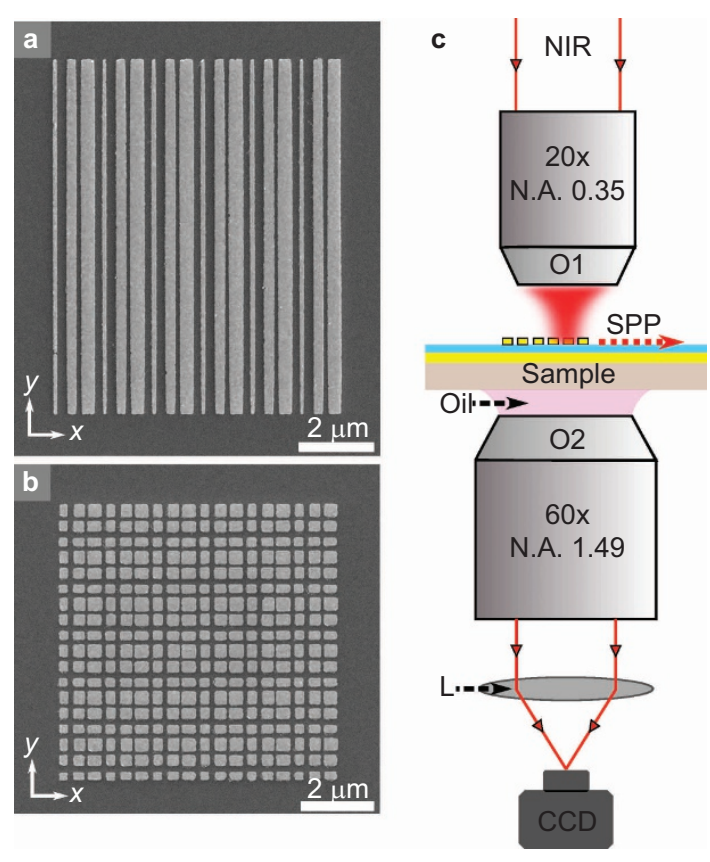

Figure 3 Optical characterization of plasmonic couplers. (a, $\mathbf{b}$ ) SEM images of (a) fabricated 10- $\mu \mathrm{m}$-long 1D-periodic SPP coupler composed of 6 super cells with period and strip widths taken from the optimized design, and (b) 2D-periodic polarization-sensitive SPP coupler composed of $6 \times 6$ super cells (Figure 2c). (c) Schematic of the experimental set-up for leakage radiation microscopy. An incident polarized and collimated beam from a fiber-coupled tunable laser source (1500$1620 \mathrm{~nm}$ ) is focused onto the sample by an objective (O1). Upon SPP excitation, leakage radiation from the sample plane is collected with an oil-immersion objective (O2) and imaged by a tube-lens ( $\mathrm{L}$ ) onto a cooled IR-CCD camera positioned at the microscope conjugate image plane. 1D, one-dimensional; 2D, two-dimensional; SEM, scanning electron microscopy; SPP, surface plasmon polariton.

We believe the discrepancy is related to fabrication imperfections due to proximity effects and liftoff, different excitation conditions, and additional losses associated with scattering and grain boundary effects in thin films as well as the increased damping related to 3-nm-thin Ti adhesion layers between gold/glass interfaces. ${ }^{24}$ The last parameter, the directivity, is experimentally determined by taking the ratio of the integrated pixel intensities in areas of $10 \mu \mathrm{m} \times 20 \mu \mathrm{m}$ centred $20 \mu \mathrm{m}$ to the left and right of $x_{0}$, respectively. In this way, we obtain a directivity of $\sim 675 \pm 160$, where the quite high uncertainty relates to a strong sensitivity towards beam positioning. As an additional comment, it should be stressed that the fabricated $1 \mathrm{D}$-periodic SPP coupler is not particularly sensitive to the design wavelength, demonstrating relatively high coupling efficiencies $(C>19 \%)$ and directivities $(D>43)$ in the entire wavelength range 1500-1600 nm (Supplementary Fig. S1).

The 2D-periodic SPP coupler (Figure 3b) has been characterized in the same way as above, but for both $x$ - and $y$-polarizations of the incident light in order to verify the polarization-controlled excitation of SPPs. From LRM images (Figure 4c and 4d), it is indeed seen that the orthogonal linear polarizations excite SPPs propagating in orthogonal directions with a high directivity. In particular, we obtain for both polarizations the coupling efficiencies of $\sim 25 \%$ with directivities $D>200$. One should note that despite the noticeable difference in directivities evaluated for orthogonal polarizations (Fig. 4c and 4d), which might be related to slight asymmetry in the fabricated SPP couplers (Figure 3b), this difference has no practical importance since, for both polarizations, practically all the SPP power (>99\%) flows in the direction dictated by the linear phase gradient. As a final comment, 

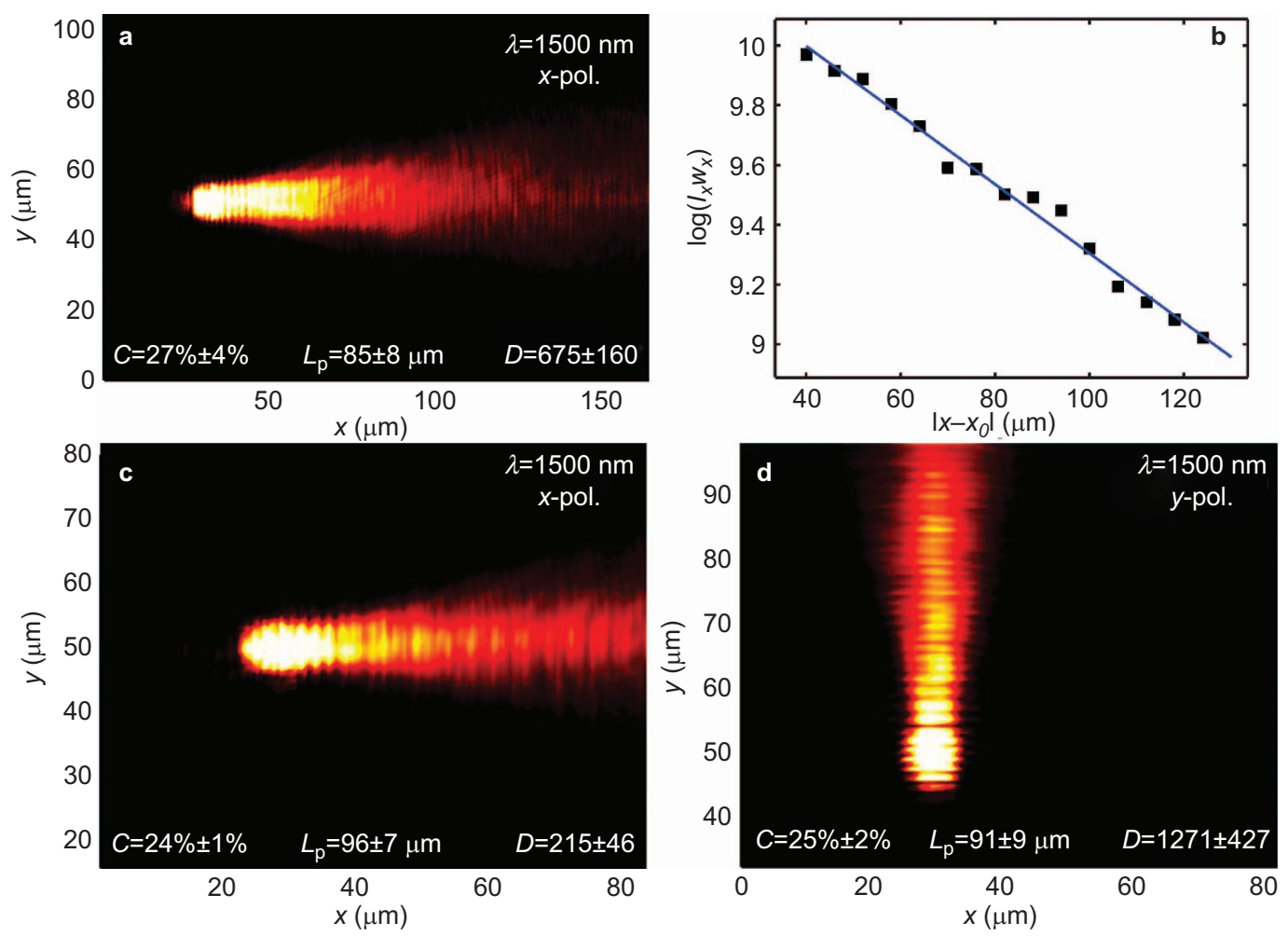

Figure 4 Efficient unidirectional polarization-controlled SPP excitation. Optical characterization of (a, b) 1D-periodic SPP coupler (Figure 3a) and (c, d) 2D-periodic polarization-controlled SPP coupler (Figure 3b). (a, c, d) Recorded LRM images for a free-space wavelength of $1500 \mathrm{~nm}$. For each image, the coupling efficiency, $C$, propagation length, $L_{\mathrm{p}}$, and directivity, $D$, are indicated. The input beam is in each case positioned to optimize the coupling efficiency and directivity. (b) Variation of the product of SPP intensity and beam waist, $I_{x} w_{x}$, on a logarithmic scale as a function of the distance $u=\left|x-x_{0}\right|$, where $x_{0}$ is the excitation spot $x$-coordinate. The first order polynomial fitted to the data points (blue curve) is used to evaluate the coupling efficiency and the propagation length. 1D, one-dimensional; 2D, two-dimensional; LRM, leakage radiation microscopy; SPP, surface plasmon polariton.

we emphasize that the 2D-periodic polarization-controlled SPP coupler also demonstrates reasonable performance in the wavelength range 1500-1600 nm ( $C>15 \%$ and $D>44$, cf. Supplementary Fig. S2).

\section{CONCLUSIONS}

We have illustrated with the examples in the previous section how GSP-based gradient metasurfaces can be designed at telecom wavelengths for efficient and unidirectional excitation of SPPs, with the added possibility to control the direction of propagation via the linear polarization of the incident beam. The latter functionality is associated with 2D-periodic birefringent metasurfaces resulting in orthogonal reflection-phase gradients for orthogonal polarizations. ${ }^{21}$ In this work, we designed the GSP resonators from a reference structure consisting of a continuous glass spacer on top of the gold film, thereby allowing us to fabricate the appropriate nanostructures by only depositing gold in the resist profile. It should, however, be noted that the design approach can equally well be applied to the configuration, in which the dielectric spacer, such as glass, is located only under the gold strips and nanobricks (Supplementary Figs. S3-S6). The fabrication of these SPP couplers requires deposition of both glass and gold, which makes it more difficult to reach the desired geometrical dimensions due to inclined side walls. ${ }^{25}$ The advantage, on the other hand, is the possibility of excitation of SPPs propagating along a gold-air interface (open to external perturbations that is important, for example, in sensing applications) over long distances, e.g., exceeding $200 \mu \mathrm{m}$ at $\lambda=1500 \mathrm{~nm}$ (Supplementary Fig. S6). Finally, it should be mentioned that, using extensive numerical simulations, we have also confirmed that the GSP-based gradient metasurfaces perform indeed significantly better than those without phase gradient, i.e., regular GSPbased grating couplers, with respect to both efficiency and directivity of the SPP excitation (Supplementary Fig. S7).

The intriguing result that a single super cell of the 2D-periodic SPP coupler allows for unidirectional polarization-controlled excitation of SPPs (Figure 2d) demonstrates a new class of optical elements, which are wavelength-sized polarization-sensitive scatterers. We name these elements metascatterers, and exemplify their usefulness in compact plasmonic circuits by verifying efficient and polarization-controlled excitation of SPPs using a GSP-based metascatterer with glass layer located only underneath the nanobricks (Figure 5a). Using a Gaussian input beam with the spot size $w_{0} \cong 1 \mu \mathrm{m}$, we obtained LRM images that clearly verify the polarization-controlled unidirectional excitation of SPPs (Figure $5 b$ and $5 c$ ), with more quantitative analysis of the images revealing an appreciable coupling efficiency of about $4 \%-8 \%$ and directivity of $\sim 6$. It is to be noted that, in this case, we had to use a very tightly confined incident beam in order to increase its interaction with the micron-sized metascatterer. As a result, the incident field was very inhomogeneous in both phase and amplitude distributions across the metascatterer, leading thereby to a decreased coupling efficiency.

We believe that the efficient unidirectional polarization-controlled excitation of SPPs, using either individual metascatterers or their periodic arrays, opens a unique possibility to simultaneously achieve both high SPP excitation efficiency and acceptance of any polarization of incident light that can be exploited for the realization of polarization-independent plasmonic components, an important milestone in the development of 

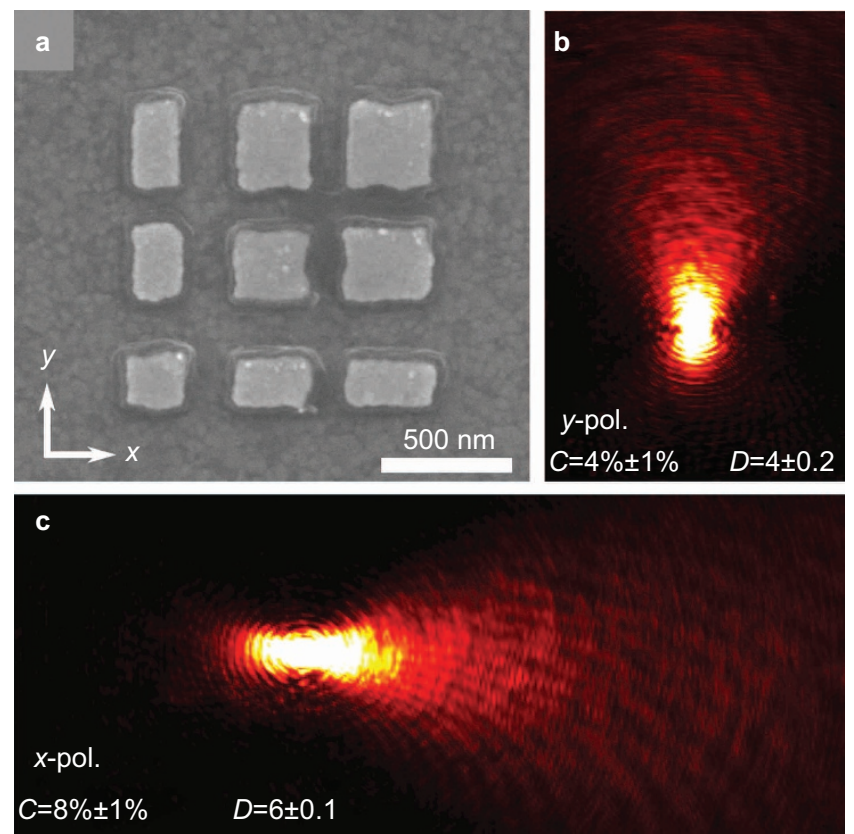

Figure 5 Plasmonic metascatterer. (a) SEM image of a metascatterer composed of nine GSP resonators arranged in a $3 \times 3$ array. The $50-n m$-thin glass spacer is located only below the 50-nm-thick gold nanobricks. The lower gold film is $80 \mathrm{~nm}$ thick. (b, c) LRM-images showing SPP excitation along (b) $y$-direction for $y$-polarized light and (c) $x$-direction for $x$-polarized light when $\lambda=1500 \mathrm{~nm}$. Due to the small size of the metascatterer, the spot size of the incident Gaussian beam is reduced to $w_{0}=1 \mu \mathrm{m}$. GSP, gap surface plasmon; LRM, leakage radiation microscopy; SEM, scanning electron microscopy; SPP, surface plasmon polariton.

integrated plasmonic circuits. The developed approach represents also a new way of encoding the polarization information in SPPs, suggesting the efficient method for inter-conversion of polarization-encoded photon qubits and SPPs, a process that is very important in the context of quantum plasmonic circuits. ${ }^{26}$ Another interesting development can be to realize complete on-chip characterization of polarization states of light by combining the polarization-controlled SPP coupler, which allows one to excite separate SPPs associated with orthogonal linear polarizations, with a suitable plasmonic interference circuitry ${ }^{27}$ to compare their phases and integrated SPP detectors ${ }^{28}$ to conduct quantitative analysis of amplitudes of the incident orthogonal linear polarizations as well as their phase difference. Overall, we foresee many exciting developments and applications of the demonstrated approach within plasmonics.

\section{ACKNOWLEDGEMENTS}

The authors would like to acknowledge the financial support from the Danish Council for Independent Research (the FTP project ANAP, contract no. 09072949, and the FNU project, contract no. 12-124690).

1 Barnes C, Dereux A, Ebbesen TW. Surface plasmon subwavelength optics. Nature 2003; 424: 824-830.
2 Anker JN, Hall WP, Lyandres 0 , Shah NC, Xhao J et al. Biosensing with plasmonics nanosensors. Nat Mater 2008; 7: 442-453.

3 Tame MS, McEnery KR, Özdemir ŞK, Lee J, Maier SA et al. Quantum plasmonics. Nat Phys 2013; 9: 329-340.

4 Gramotnev DK, Bozhevolnyi SI. Plasmonics beyond the diffraction limit. Nat Photon 2010; 4: 83-91.

5 Atwater HA, Polman A. Plasmonics for improved photovoltaic devices. Nat Mater 2010; 9: 205-213.

6 Lopez-Tejeira F, Rodrigo SG, Martín-Moreno L, García-Vidal FJ, Devaux E et al. Efficient unidirectional nanoslit couplers for surface plasmons. Nat Phys 2007; 3 . 324-328.

7 Radko IP, Bozhevolnyi SI, Brucoli G, Martín-Moreno L, García-Vidal FJ et al. Efficient unidirectional ridge excitation of surface plasmons. Opt Express 2009; 17: 7228 7232.

8 Baron A, Devaux E, Rodier JC, Hugonin JP, Rousseau E et al. Compact antenna for efficient and unidirectional launching and decoupling of surface plasmons. Nano Lett 2011; 11: 4207-4212.

9 Li X, Tan Q, Bai B, Jin G. Experimental demonstration of tunable directional excitation of surface plasmon polaritons with a subwavelength metallic double slit. Appl Phys Lett 2011; 98: 251109.

10 Sonnefraud Y, Kerman S, Di Martino G, Lei DY, Maier SA. Directional excitation of surface plasmon polaritons via nanoslits under varied incidence observed using leakage radiation microscopy. Opt Express 2012; 20: 4893-4902.

11 Yu N, Genevet P, Kats MA, Aieta F, Tetienne JP et al. Light propagation with phase discontinuities: generalized laws of reflection and refraction. Science $2011 ; 334$ 333-337.

12 Kildishev AV, Boltasseva A, Shalaev VM. Planar photonics with metasurfaces. Science 2013; 339: 1232009.

13 Sun S, He Q, Xiao S, Xu Q, Li X et al. Gradient-index meta-surfaces as a bridge linking propagating waves and surface waves. Nat Mater 2012; 11: 426-430.

14 Qu C, Xiao S, Sun S, He Q, Zhou L. A theoretical study on the conversion efficiencies of gradient meta-surfaces. EPL 2013; 101: 54002.

15 Lin J, Mueller JPB, Wang Q, Yuan G, Antoniou N et al. Polarization-controlled tunable directional coupling of surface plasmon polaritons. Science 2013; 340: 331-334.

16 Huang L, Chen X, Bai B, Tan Q, Jin G et al. Helicity dependent directional surface plasmon polaritons excitation using a metasurface with interfacial phase discontinuity. Light Sci App/2013; 2: e70. doi:10.1038/lsa.2013.26

17 Sun S, Yang KY, Wang CM, Juan TK, Chen WT et al. High-efficiency broadband anomalous reflection by gradient meta-surfaces. Nano Lett 2012; 12: 6223-6229.

$18 \mathrm{LiX}$, Xiao S, Cai B, He Q, Cui TJ et al. Flat metasurfaces to focus electromagnetic waves in reflection geometry. Opt Lett 2012; 37: 4940-4942.

19 Pors A, Nielsen MG, Eriksen RL, Bozhevolnyi SI. Broadband focusing mirrors based on plasmonic gradient metasurfaces. Nano Lett 2013; 13: 829-834.

20 Liu Y, Palomba S, Park Y, Zentgraf T, Yin X et al. Compact magnetic antennas for directional excitation of surface plasmons. Nano Lett 2012; 12: 4853-4858.

21 Pors A, Albrektsen O, Radko IP, Bozhevolnyi SI. Gap plasmon-based metasurfaces for total control of reflected light. Sci Rep 2013; 3: 2155.

22 Nielsen MG, Gramotnev DK, Pors A, Albrektsen O, Bozhevolnyi SI. Continuous layer gap plasmon resonators. Opt Express 2011; 19: 19310-19322.

23 Larouche S, Smith DR. Reconciliation of generalized refraction with diffraction theory. Opt Lett 2012; 37: 2391-2393.

24 Pors A, Nielsen MG, Bozhevolnyi SI. Broadband plasmonic half-wave plates in reflection. Opt Lett 2013; 38: 513-515.

25 Yuan HK, Chettiar UK, Cai W, Kildishev AV, Boltasseva A et al. A negative permeability material at red light. Opt Express 2007; 15: 1076-1083.

26 de Leon NP, Lukin MD, Park H. Quantum plasmonic circuits. J Sel Topics Quantum Electron 2012; 18: 1781-1791.

27 Ditlbacher H, Krenn JR, Schider G, Leitner A, Aussenegg FR. Two-dimensional optics with surface plasmon polaritons. Appl Phys Lett 2002; 81: 1762-1764.

28 Genevet P, Lin J, Kats MA, Capasso F. Holographic detection of the orbital angular momentum of light with plasmonic photodiodes. Nat Commun 2012; 3: 1278.

This work is licensed under a Creative Commons Attribution-

NonCommercial-NoDerivs 3.0 Unported License. The images or other third arty material in this article are included in the article's Creative Commons license, unles indicated otherwise in the credit line; if the material is not included under the Creative Commons license, users will need to obtain permission from the license holder to reproduce the material. To view a copy of this license, visit http://creativecommons.org/licenses/ by-nc-nd/3.0/ 